\section{HIGHLY BIOCOMPATIBLE RESTORATIONS}

Glass carbomer materials from GCP Dental is an exclusive development in dental materials technology providing long lasting, strong, highly biocompatible restorations replicating the tooth structure.

Glass Carbomer is the result of over a decade of university research from teams at ACTA (Amsterdam) and Queen Mary University, London. Their objective was to develop materials which "would allow the tooth to regenerate itself to the original element or better' without the side effects of any potentially toxic content. Glass carbomer uses biomimetic natural remineralising processes provided by minerals released from the hydroxyapatite containing fluoride aluminium silicate glass. The result is a restoration with high compressive and flexural strength, plus strong wear and acid resistance. The capsule activated materials are now available for long term restorations and core build up, fissure sealing, and crown and bridge cementation.

Further information and supporting clinical evidence is available at www.gcp-dental.com or call Plandent on 0500500322 to arrange a demonstration.

\title{
A NEW, EFFECTIVE THERAPEUTIC ENDODONTIC DRESSING
}

ODONTOPASTE is a new and effective therapeutic endodontic dressing available now from Henry Schein Dental. The real alternative to Ledermix, ODONTOPASTE is a zincoxide-based paste, used to temporarily obturate root canals and is highly effective in helping reduce bacterial contamination and post-operative pain following endodontic therapy.

The pain associated with endodontic procedures is often the result of inflammation of the pulp and/or periapical tissues. ODONTOPASTE tackles this pain thanks to the antiinflammatory characteristics of its ingredients, clindamycin hydrochloride, a broad-spectrum antibiotic, and triamcinolone acetonide - a steroid-based anti-inflammatory agent, which acts through direct contact with the inflamed tissue. The majority of these active components are released within the first few days following application, therefore ensuring rapid action and fast pain relief for the patient.
ODONTOPASTE is supplied in an easy-to-use, premixed $8 \mathrm{~g}$ tube and applied directly into the inflamed pulp with a cotton pellet or into the root canal using a lenticulospiral instrument. As ODONTOPASTE is water soluble it can easily be rinsed out, leaving the prepared canal available for the dentist to obturate using their material of choice.

\section{ODONTOPASTE is a Class III Medical Device} whereby Ledermix is a POM Pharmaceutical, therefore ODONTOPASTE is not a direct comparison but an alternative treatment option.

www.henryschein.co.uk

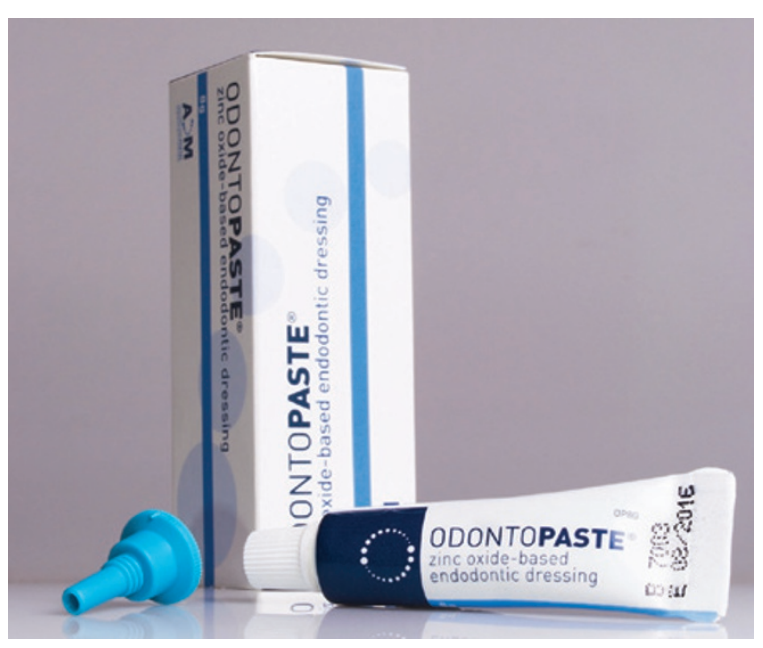

\section{SIGNIFICANTLY INCREASING PRODUCTIVITY}

Fully anatomical restorations can now be made even quicker and with greater colour accuracy. Sirona Dental Systems now offers preshaded translucent zirconium oxide. inCoris TZI C is available in ten classic tooth colours and in three different block sizes. The user can save time by using the pre-shaded ceramic blocks, as the restorations no longer need to be dipped and dried in colouring liquid. Another advantage is that since the block is a solid colour all the way through, the user can avoid creating light areas when grinding.

The new zirconium oxide blocks are ideal for the production of full crowns and fully anatomical bridges with the inLab and CEREC production units. inCoris TZI C is classically sintered and can be finalised with commercial paints and gloss paints. The inFire HTC speed sintering furnace from Sirona quickly sinters inCoris TZI $\mathrm{C}$ in 60 minutes, as it does with all of Sirona's other zirconium oxides (inCoris ZI and inCoris TZI).

The simple workflow also makes pre-shaded translucent zirconium oxide interesting for the dentist simple crown and bridge work can be carried out chairside with the CEREC system. The elimination of two work steps (drying and dipping) saves around 30 minutes and can significantly increase productivity. The zirconium oxide restorations can also be classically cemented.

Call Sirona Dental Systems on 08450715040 or emailinfo@ sironadental.co.uk.

\section{LONG-LASTING ANTIMICROBIAL ACTION}

Gum health through effective plaque control is just one of the many beneficial features of Oral-B's ProExpert toothpaste and it's the inclusion of stabilised stannous fluoride $\left(\mathrm{SnF}_{2}\right)$ that makes the difference.

This powerful ingredient gives Oral-B's Pro-Expert toothpaste a long-lasting antimicrobial action which fights plaque and consequently gum problems. Laboratory and clinical studies have confirmed that the inclusion of $\mathrm{SnF}_{2}$ will inhibit antimicrobial growth as well as reducing the ability of bacteria to 'stick' to tooth and gum surfaces.

The gum health benefits do not stop there, thanks to the inclusion of sodium hexametaphosphate. This protects against calculus formation and staining, thereby reducing a further cause of plaque retention on the roughened surface of the calculus. The combined force of these two plaque reducing agents help protect against gum problems.

Whilst the inclusion of fluoride is a given in toothpaste, to afford maximum protection, a formulation must contain other proven ingredients that work in harmony to promote good oral health. 